\title{
Le Kosovo en voie d'homogénéisation : quelle est la part du « nettoyage ethnique »?
}

Kosovo towards homogeneity: how much is due to "ethnic cleansing"?

Der Kosovo auf dem Wege der Homogenisierung : Welches ist der Anteil der "ethnischen Säuberung"?

\section{Michel Roux}

\section{(2) OpenEdition}

\section{Journals}

Édition électronique

URL : http://journals.openedition.org/rge/572

DOI : $10.4000 /$ rge. 572

ISSN : 2108-6478

Éditeur

Association des géographes de l'Est

Édition imprimée

Date de publication : 1 janvier 2005

Pagination : 23-33

ISSN : 0035-3213

Référence électronique

Michel Roux, « Le Kosovo en voie d'homogénéisation : quelle est la part du «nettoyage ethnique » ?», Revue Géographique de l'Est [En ligne], vol. 45 / 1 | 2005, mis en ligne le 19 mai 2009, consulté le 08 septembre 2020. URL : http://journals.openedition.org/rge/572 ; DOI : https://doi.org/10.4000/rge.572

Ce document a été généré automatiquement le 8 septembre 2020.

Tous droits réservés 


\title{
Le Kosovo en voie
}

\section{d'homogénéisation : quelle est la part du « nettoyage ethnique »?}

\author{
Kosovo towards homogeneity: how much is due to "ethnic cleansing"? \\ Der Kosovo auf dem Wege der Homogenisierung: Welches ist der Anteil der \\ «ethnischen Säuberung»?
}

Michel Roux

1 L'histoire du Kosovo ${ }^{1}$ au XX $X^{e}$ siècle recèle un paradoxe. Alors que cette région conquise sur l'Empire ottoman en 1912 est demeurée ensuite dans le cadre de la Serbie et du Monténégro, puis des Yougoslavie successives (sauf de 1941 et 1945) et sous le contrôle effectif de leurs gouvernements (sauf de 1915 à 1918 et depuis juin 1999), Serbes et Monténégrins ont vu leur part de sa population diminuer jusqu'à être réduite, depuis 1999, à une minorité de quelque $5 \%$, implantée aux marges nord et dans quelques enclaves, face à une majorité albanaise aujourd'hui massivement acquise à l'idée d'indépendance. Cette régression de leur part dans la population totale, qui n'est pas continue au cours du siècle, a été très sensible entre les recensements de 1961 et de 1991, très brutale à l'été 1999. Selon les périodes, elle s'explique dans des proportions variables par des facteurs politiques, démographiques et économiques. Les dirigeants, les intellectuels et les médias serbes en orientent l'interprétation de manière à ériger le « nettoyage ethnique » en explication centrale dans le cadre d'une vision victimiste de l'histoire. Du côté albanais, on récuse cette vision des choses mais on construit une interprétation du même genre en tirant argument de ses propres déboires. Le présent article, après un bref rappel historique, entend analyser cette surenchère à propos du «nettoyage ethnique » et faire le point sur la situation présente du peuplement du Kosovo, notamment sur la situation des Serbes et des Monténégrins². 


\section{Bref rappel du contexte politique}

2 La Serbie et le Monténégro ont conquis le Kosovo en 1912 au cours de la première guerre balkanique où ils s'étaient joints, pour combattre l'Empire ottoman, à la Grèce et à la Bulgarie (laquelle se retourna contre ses alliés immédiatement après, en vain, lors de la seconde guerre balkanique). La Conférence de Londres (décembre 1912-mai 1913), qui reconnut aussi l'indépendance de l'Albanie et en fixa les frontières, entérina la manière dont ils s'étaient partagé le Kosovo. En effet, bien que les Albanais y fussent majoritaires ${ }^{3}$, les puissances étaient sensibles à l'argument historique serbe (le Kosovo, cœur du royaume serbe aux XIII-XIV ${ }^{e}$ siècles) et, doutant de la capacité des Albanais à assumer leur indépendance, elles inclinaient à ne la leur accorder que sur des territoires qu'ils peuplaient quasi-exclusivement (Gravier, 1913). Mais cette configuration ne dura guère car de 1915 à 1918, au cours de la Première Guerre mondiale, le Kosovo fut sous occupation austro-bulgare. Sous le Royaume des Serbes, Croates et Slovènes, créé en 1918 et rebaptisé en 1929 Royaume de Yougoslavie, le Kosovo n'a jamais été une subdivision de l'État. Celui-ci l'a délibérément partagé entre plusieurs oblasts, puis en plusieurs banovines, de manière à fragmenter l'aire de peuplement majoritairement albanais. Pendant la Seconde Guerre mondiale, après le démembrement de la Yougoslavie, la plus grande partie du Kosovo est rattachée à la "Grande Albanie» promue par Mussolini, tandis que le nord, avec les mines de Mitrovica, fait partie de la Serbie sous occupation allemande et que la partie orientale est donnée à la Bulgarie.

3 C'est en 1945 que le Kosovo est érigé en territoire autonome, plus tard rebaptisé province autonome en étant aligné sur le statut de la Voïvodine. Cette autonomie, d'abord très réduite, ne sera accrue que par des amendements constitutionnels votés à partir de 1969 et repris dans la Constitution fédérale de 1974. Celle-ci définit le Kosovo (comme la Voïvodine) à la fois comme province autonome de la République de Serbie et, au même titre que celle-ci, comme l'une des huit composantes de l'État fédéral. Cette étrange disposition fut considérée par les Serbes comme destinée à affaiblir leur république (la seule à comporter des provinces autonomes), mais l'autonomie accrue ne suffisait pas aux Albanais, qui, après la mort de Tito, manifestèrent en 1981, contestant leur statut de minoritét et réclamant l'élévation du Kosovo au rang de septième république yougoslave ${ }^{5}$. Suit une dure répression policière et judiciaire qui contribue à détériorer les rapports entre Albanais et Serbes dans la région. Le contexte yougoslave est alors globalement défavorable : stagnation économique, montée du chômage et des difficultés sociales, mésententes entre républiques, renforcement des courants nationalistes et «leadership collectif faible», selon l'expression de Renéo Lukic (Lukic, 2003, p. 71).

4 Slobodan Milošević, arrivé au pouvoir en Serbie en 1987, en réforme deux ans plus tard la Constitution, supprimant pratiquement toute autonomie politique des provinces. Débute alors la marginalisation des Albanais, dont l'élite avait commencé à accéder au pouvoir provincial et communal et aux responsabilités économiques et culturelles vers 1970 : licenciements massifs dans le secteur public, mise à pied de la plus grande partie du corps enseignant par suite de conflits sur la réforme des programmes scolaires. Cette époque est aussi celle où, après la chute du mur de Berlin, la majorité des États communistes, dont la Yougoslavie, autorisent le pluripartisme. Dès lors se fondent de nouveaux partis à électorat le plus souvent «ethnique ", dont, chez les Albanais, la 
Ligue démocratique du Kosovo (LDK). En 1991, la sécession de la Slovénie et de la Croatie change les perspectives des Albanais, jusque là plutôt disposés à demeurer dans le cadre de la Yougoslavie sous réserve d'y retrouver l'autonomie. D'où, à la suite d'un vote massif en faveur de l'indépendance lors d'un référendum clandestin, la proclamation de celle-ci (octobre). En même temps se mettent en place des institutions parallèles: embryon de gouvernement, écoles et centres de soins financés par les contributions des Albanais locaux ou émigrés. Le gouvernement de la Serbie réprime sans réellement chercher à éradiquer ces institutions illégales relevant d'une résistance pacifique. Car les Serbes sont impliqués dans la guerre en Croatie et en Bosnie. Au Kosovo, de part et d'autre, on gagne du temps : à la Serbie est épargnée l'ouverture d'un "front sud», aux Kosovars un «nettoyage ethnique » massif que les dirigeants de la LDK estiment inévitable en cas de révolte armée.

5 La donne change après la signature des accords de Dayton, auxquels Milošević a accepté de participer avec pour condition tacite la non prise en compte de la question du Kosovo. Dès lors, les Albanais se sentent floués, une opposition plus radicale s'exprime autour de l'Armée de libération du Kosovo (UÇK), cela aboutira en 1998 à la guerre entre cette armée improvisée et les forces serbes puis, sous pression internationale, à l'arrêt des combats et à la mise en place d'une mission d'observation de l'OSCE, l'année suivante à la reprise des combats, à l'échec des négociations de Rambouillet, aux bombardements de l'OTAN, à la tentative de Belgrade d'expulser en masse les Albanais du Kosovo, à l'imposition d'une tutelle internationale sur la province, au retour des Albanais, à la fuite de nombreux Serbes et d'autres minoritaires (exposé détaillé des faits dans Hubrecht, 2001).

En résumé, le Kosovo a été très secoué au $\mathrm{XX}^{\mathrm{e}}$ siècle. Il a vécu des conflits armés pendant une vingtaine d'années et connu huit situations géopolitiques successives : 1 ) dans l'Empire ottoman ; 2) partagé entre Serbie et Monténégro ; 3) sous occupation austro-bulgare ; 4) dans la Yougoslavie monarchique ; 5) dans la «Grande Albanie »;6) dans la Yougoslavie communiste, avec quatre statuts constitutionnels successifs ; 7) dans la Yougoslavie, un peu plus tard réduite à l'association Serbie-Monténégro, avec un régime d'apartheid de facto ; 8) formellement dans ce dernier État, mais sous tutelle internationale provisoire et sans perspective statutaire au moment où ces lignes sont écrites.

7 Parallèlement, le Kosovo a vécu des changements démographiques, culturels, sociaux et économiques très rapides, surtout après la Seconde Guerre mondiale : la population triple presque malgré un solde migratoire négatif ; les Albanais, analphabètes à $90 \%$ en 1945, bénéficient sous le régime communiste de la scolarité obligatoire dans leur langue, ce qui fait émerger une élite locale étoffée, d'autant plus qu'en 1970 l'Université de Priština est mise en place et attire aussi des Albanais de Macédoine ; l'urbanisation et l'industrialisation sont dynamiques grâce aux financements fédéraux (sans toutefois pouvoir faire progresser l'emploi de façon suffisante face à l'explosion démographique). Dans ce contexte, des valeurs et des représentations à la fois claniques, confessionnelles, nationalistes, marxistes, libérales, se bousculent dans les têtes, s'expriment ou sont tues. On peut illustrer le raccourci que vit cette région en évoquant la scène suivante : au début des années 1990, face au durcissement de la politique de Belgrade à leur égard, les Albanais décident de « resserrer les rangs » dans une optique nationaliste. Pour cela, ils tiennent une assemblée destinée à éteindre les vendettas en cours parmi eux au titre du Kanun, leur code coutumier médiéval. Celui qui préside 
cette assemblée, spécialiste reconnu de cette tradition, est aussi professeur de français à l'Université...

8 Pendant toute cette période, l'un des enjeux principaux, pas nécessairement explicité, est la question de savoir si le Kosovo sera finalement serbe ou albanais, ou si l'on saura dépasser cette alternative par une intégration réussie. Étudier les fluctuations de la composition de sa population et les explications qui en sont données est, de ce point de vue, instructif.

\section{Serbes et Albanais au Kosovo depuis 1912 : données numériques}

9 Étudier la dynamique de la population du Kosovo comporte des incertitudes majeures en début et en fin de période. En 1913, le Kosovo est crédité de 497000 habitants $^{6}$, soit 58000 de plus qu'au recensement de 1921, mais il est impossible de déterminer si Serbes et Monténégrins représentaient alors le tiers ou le cinquième de cette population, tant les sources sont contradictoires sur les pertes de guerre et les migrations forcées des années suivantes. En 1991, bien que les Albanais aient boycotté le recensement, les services statistiques ont produit une estimation de leur nombre qui peut être considérée comme fiable. Mais, passé cette date, il n'y a plus eu de dénombrement, et l'enregistrement défectueux des naissances et décès, les incertitudes qui pèsent sur l'estimation des mouvements migratoires et la diffusion d'informations fausses aboutissent au flou le plus total. On examinera donc la période où les données officielles sont les moins incertaines, 1921-1991 (tableau 1), tout en soulignant que les sources courantes abondent en raisonnements biaisés et en falsifications ${ }^{7}$.

Tableau 1 : Évolution de la population serbo-monténégrine et albanaise du Kosovo, 1921-1991.

\begin{tabular}{|c|c|c|c|c|c|c|c|c|}
\hline & & & Serbes e & t Montér & égrins & Albanais & & \\
\hline Année & Pop. totale (milliers) & AAM (\%o) & milliers & AAM & $\%$ & milliers & $A A M$ & $\%$ \\
\hline 1921 & 439 & & 93 & & 21,2 & 280 & & 63,8 \\
\hline 1931 & 552 & 23,2 & 151 & 49,7 & 27,4 & 332 & 17,2 & 60,1 \\
\hline 1939 & 645 & 19,7 & 222 & 49,4 & 34,4 & 370 à 380 & 13,6 à 17,0 & 57,3 à 58,9 \\
\hline 1948 & 734 & 14,5 & 200 & $-12,5$ & 27,2 & 498 & 30,5 à 33,6 & 68,5 \\
\hline 1953 & 808 & 19,4 & 221 & 20,2 & 27,4 & 525 & 10,6 & 64,9 \\
\hline 1961 & 964 & 22,3 & 265 & 23,0 & 27,4 & 647 & 26,5 & 67,2 \\
\hline 1971 & 1244 & 25,8 & 260 & $-1,9$ & 20,9 & 916 & 35,4 & 73,7 \\
\hline 1981 & 1585 & 24,5 & 237 & $-9,2$ & 14,9 & 1227 & 29,7 & 77,4 \\
\hline
\end{tabular}




\begin{tabular}{|l|l|l|l|l|l|l|l|l|}
\hline 1991 & 1955 & 21,2 & 215 & $-9,7$ & 11,0 & 1608 & 27,4 & 82,2 \\
\hline
\end{tabular}

AAM : accroissement annuel moyen au cours de la période précédente.

Avant guerre, Serbes et Monténégrins ont été recensés comme orthodoxes en 1921 et 1931, comme slaves en 1939 (catégorie excluant les musulmans), les Albanais comme albanophones en 1921. Pour 1939 j'ai estimé leur nombre à partir de celui des non-slaves.

Sources : Recensements de la population, sauf 1939, dénombrement administratif cité par M. Obradović, 1981, p. 223.

Les données du tableau 1 font apparaître une forte croissance du nombre de Serbes et Monténégrins et de leur part dans la population jusqu'à la veille de la Seconde Guerre mondiale, un recul pendant celle-ci, puis une progression plus modeste en nombre assortie d'une stabilité en pourcentage, enfin une baisse après 1961. Celle-ci, contemporaine du pic de l'explosion démographique albanaise, les réduit en 1991 à $11 \%$ d'une population qui, depuis 1921, a plus que quadruplé.

11 Précisons d'abord que comparer les neuf sources utilisées pose des problèmes, car elles diffèrent par le degré de fiabilité, la méthodologie et le contexte politique (Roux, 2000). Ainsi, il est clair que le recensement de 1921 n'a pu être effectué dans des conditions normales dans une région en partie insurgée d'un État nouveau-né. D'autre part, le recours successif à des dénominations confessionnelles, ethnolinguistiques puis nationales rend la comparabilité des données plus incertaine. Enfin, c'est le contexte politique qui explique la faible progression du nombre d'Albanais recensés entre 1948 et 1953 : la Yougoslavie ayant conclu un accord d'échange de populations avec la Turquie, des Albanais se sont déclarés Turcs (1 300 Turcs au Kosovo en 1948, 34600 en 1953), ce à quoi les autorités les encourageaient, car les candidats au départ étaient opposés au régime. Mais on peut soupçonner aussi qu'en 1948 le nombre d'Albanais avait été surestimé, celui des Turcs sous-estimé, ces oscillations renvoyant à la fois à des pressions politiques et à une ambivalence identitaire, assez fréquente à date ancienne et dérangeante pour les nationalistes. Ce qui le suggère, c'est qu'au recensement de 1953, parmi les habitants du Kosovo 25100 ont déclaré avoir le turc pour langue maternelle. Pourtant, certains auteurs préfèrent considérer que tous ceux qui ont été recensés en 1948 comme Albanais l'étaient réellement, car cela nourrit l'idée que la forte croissance de leur nombre depuis 1939 s'explique par une importante immigration depuis l'Albanie, pendant la Seconde Guerre mondiale et jusqu'à la rupture Tito-Staline. Si réellement 75000 Albanais d'Albanie ont obtenu la citoyenneté yougoslave (Bataković, 2000, p. 211), cela renvoie à d'autres incertitudes et polémiques sur les pertes de guerre et les migrations de cette époque.

12 Toutefois, même si garantir l'exactitude des variations de la population totale et de ses composantes d'un recensement à l'autre est impossible, les grandes tendances lisibles sur le tableau sont incontestables. Les écarts entre les taux de croissance des populations serbo-monténégrine et albanaise d'une part, les taux d'accroissement naturel d'autre part (Collectif, 1957; Statistički godišnjak Jugoslavije 1955 à 1991), permettent d'évaluer sommairement la part des migrations dans ces processus. Entre 1921 et 1939, la croissance des Serbes et Monténégrins (49 p. mille par an en moyenne) a pour composante principale l'envoi au Kosovo de fonctionnaires civils et militaires du nouvel État, et plus encore d'au moins 60000 paysans installés au titre de la réforme agraire sur des terres domaniales ou sur des terres confisquées à des Albanais (Obradović, 1981). Chez ceux-ci, un taux de croissance de $11 \%$ seulement, bien 
inférieur à leur accroissement naturel, traduit l'effet d'une émigration vers la Turquie et l'Albanie (rebelles en fuite, paysans expropriés...). Pendant la Seconde Guerre mondiale, le départ des fonctionnaires et l'expulsion des colons agraires de l'entre deux guerres font chuter le nombre de Serbes et de Monténégrins, ce dont le recensement de 1948 porte encore la trace bien que les retours soient déjà largement entamés - même si le nouveau régime a interdit en 1945 à la majorité des colons de revenir pour ne pas accroître les tensions intercommunautaires et pour complaire à l'Albanie, alors son alliée (Bataković, 2000, p. 210).

13 Jusqu'au début des années 1960, Serbes et Monténégrins maintiennent leur part dans la population du Kosovo car leur accroissement naturel est encore fort, de nombreux cadres viennent s'installer dans un contexte de développement économique et des Albanais quittent le pays sous l'étiquette de Turcs. Mais l'émigration économique des Serbes et Monténégrins du Kosovo débute alors. En conséquence, la part de ceux qui sont nés sur place, plutôt ruraux, connaissant bien les Albanais et parfois leur langue, diminue, tandis qu'augmente celle des nouveaux venus, plutôt citadins et sans connaissance fine du contexte local.

14 Par la suite, la réduction de la part serbo-monténégrine au Kosovo va résulter à la fois de l'accroissement des départs et de la forte réduction du solde naturel, face à une population albanaise beaucoup moins mobile qui connaît dans les années 1970 le pic de son accroissement naturel, la mortalité baissant alors beaucoup plus vite que la natalité. Ainsi, au lieu de 60 Serbes et Monténégrins pour 100 Albanais en 1939, il n'y en a plus que 13 en 1991. Après cette date, on entre dans le flou : il est fort possible que leur part ait cessé de se réduire, voire qu'elle se soit un peu redressée. En ce sens jouent quatre arguments: redistribution de l'emploi du secteur public lors de l'éviction massive des Albanais, nouvelles tentatives d'installation d'agriculteurs (sans grand succès), hébergement de réfugiés serbes de Croatie et de Bosnie ${ }^{8}$, nombreux départs d'Albanais pour l'étranger. Toutefois, l'ambiance demeurant pénible et l'économie se dégradant au cours des années 1990, il est probable que les départs de Serbes et de Monténégrins sont restés nombreux. Quant à leur taux d'accroissement naturel, il tendait probablement vers zéro.

C'est à la fin du siècle que la violence pure redevient le principal facteur de déplacement des populations et de variation des effectifs. En 1998, les forces yougoslaves (policières, militaires et paramilitaires) aux prises avec l'armée de libération du Kosovo (UÇK) commettent des violences contre la population albanaise, chassant de leurs maisons environ 300000 personnes qui deviennent alors soit des personnes déplacées au Kosovo même, soit, pour un tiers, des réfugiés dans les pays voisins. Au printemps 1999, lors de la campagne de bombardements de l'OTAN, ce sont plus de 800000 Albanais qui sont expulsés du Kosovo par les forces yougoslaves vers l'Albanie, la Macédoine ou d'autres pays ${ }^{9}$. La plupart reviendront assez vite après le passage de la province sous la tutelle de l'ONU, mais, simultanément, au moins la moitié des Serbes, des Monténégrins et des Roms seront alors contraints de fuir, ces derniers sous l'accusation d'avoir été pour les forces yougoslaves des exécuteurs de basses œuvres. Ce renversement de situation s'inscrit dans les paysages. Ainsi au village de Sofali, près de Priština, l'armée yougoslave cantonnée à proximité brûla les maisons albanaises avant de se retirer devant les forces de l'OTAN, et quelques jours après les Albanais locaux, revenus d'exil, brûlèrent les maisons serbes dont les habitants venaient à peine de fuir. Quelques années plus tard, ce village suburbain est un vaste 
chantier, purement albanais, où les uns réparent leur maison tandis que d'autres sont installés chez des Serbes dont ils « squattent » les biens quand ils ne les ont pas achetés par le biais d'intermédiaires (transactions non enregistrées), et que d'autres encore bâtissent en toute illégalité sur le domaine public municipal.

\section{Le « nettoyage ethnique » : réalités, fantasmes et manipulations}

Certaines migrations, qui ont influé sur la composition ethno-nationale de la population, relèvent indiscutablement $d u$ "nettoyage ethnique »: outre celles qui viennent d'être évoquées, c'est le cas des expulsions violentes d'Albanais en 1912-1913, de Serbes en 1915-1918 et en 1941-44. Des mouvements moins connus relèvent de la même logique, ainsi l'exil de la modeste minorité croate soumise à des pressions serbes dans les années 1990 (Duijzings, 2000). Toutefois, dans cette matière, le passage à l'acte n'est jamais revendiqué comme tel, et l'intention l'est très rarement. La conférence de Vasa Čubrilović intitulée "L'expulsion des Albanais", donnée au Club culturel serbe de Belgrade le 7 mars 1937, en est un singulier exemple (traduction dans Grmek et al., 1993, p. 161-185). Son auteur, considérant les Albanais comme des allogènes suspects de séparatisme, dont l'implantation géographique n'est pas loin de couper la continuité du peuplement slave entre Serbie et Macédoine, estime la colonisation agraire en cours inefficace contre ce péril et suggère des méthodes radicales, susceptibles pourtant de passer inaperçues car le monde a les yeux fixés ailleurs. Il s'agit d'expulser la plus grande partie des Albanais du Kosovo, en particulier de la zone frontalière, par un mélange de pressions légales, de violences et de corruption. L'auteur reprend cette approche en octobre 1944 dans un second texte, "Le problème des minorités dans la nouvelle Yougoslavie ", en lui ajoutant un nouvel argument, celui de la collaboration de nombreux Albanais (et d'autres minoritaires) avec les occupants (extraits dans Grmek et al., 1993, p. 225-228). On retrouve des traces de cette approche du "problème albanais ", entre autres, dans le programme du Parti radical serbe diffusé lors des élections de décembre 1992. Il propose notamment d'expulser tous les Albanais immigrés d'Albanie et leur descendance et, " vu l'attitude constamment hostile de l'Albanie ", de dégager le long de la frontière une bande de 20 à $50 \mathrm{~km}$ de largeur en déplaçant toute sa population albanaise "avec compensation adéquate " (Programmes, 1993). Il est difficile d'imaginer où la Serbie aurait pu réinstaller plusieurs centaines de milliers de personnes, comment elle aurait financé une telle opération et géré les troubles qui l'auraient accompagnée. Mais le désir sous-jacent de «nettoyage ethnique " est manifeste, et le premier effet nocif d'un tel programme aura été de diffuser de telles représentations auprès de l'électorat dont ce parti espérait capter les voix.

17 Mais en pratique, il peut être malaisé d'identifier certaines initiatives comme relevant ou non du "nettoyage ethnique», et lorsque cela ne fait pas de doute, il est généralement difficile d'en évaluer la portée, dans la mesure où chaque partie en présence minimise ou nie son implication dans cette pratique et l'impute massivement à son compétiteur dans le cadre d'un discours de victimisation. Ce discours tend à légitimer les violences commises par son propre camp en les présentant comme des réactions défensives face aux violences subies. D'autre part, si on l'étudie comme source documentaire sur les pratiques du camp opposé, on y trouve trois types de 
déformations : la stigmatisation globale de l'adversaire (tous coupables), l'exagération des dégâts, l'extension de l'accusation à des domaines qui lui sont en réalité étrangers. Ce discours comporte des degrés d'élaboration différents selon qu'on a affaire au citoyen ordinaire, aux sources politiciennes et médiatiques, aux intellectuels nationalistes. Et des variations de fréquence et d'intensité selon qu'on est en période d'apaisement, de tension débutante, de tension paroxystique avec transformation du voisin en ennemi (Kullashi, 1998, chap. 9). On développera ici deux exemples pris dans le discours serbe, l'un à propos de l'émigration des Serbes et des Monténégrins du Kosovo, l'autre à propos de la natalité albanaise.

18 À partir des manifestations albanaises du printemps 1981 et de la réponse répressive des autorités se développe en Serbie un discours médiatique anti-albanais, d'abord relativement prudent (et parfois censuré), beaucoup plus agressif dès l'arrivée au pouvoir de Milošević. On chiffre alors couramment l'émigration serbo-monténégrine des deux décennies précédentes au départ du Kosovo à environ 200000 personnes, chiffres repris sans aucune critique à l'étranger ${ }^{10}, \mathrm{y}$ compris dans des publications académiques (Dragnich et Todorovich, 1984). Des observateurs américains venus à Belgrade, en décembre 1990, assister au déroulement d'élections, s'étaient même entendu demander par leurs hôtes : " les membres de la délégation savent-ils qu'au cours des vingt dernières années 400000 Serbes et Monténégrins ont quitté le Kosovo en raison de pressions ? $»^{11}$. Rappelons que Serbes et Monténégrins étaient 265000 au Kosovo en 1961, effectif en baisse de 28000 vingt ans plus tard (tableau 1). Si l'émigration nette atteint 200000 personnes, cela implique un taux d'accroissement naturel annuel moyen de $27,7 \%$. D'après les données de l'état civil, ce taux était en réalité de l'ordre de $16 \%$, ce qui permet d'évaluer l'émigration nette de ces vingt années à environ 100000 personnes. Ceci est confirmé par les données sur les « migrations ethniques intérieures par périodes » du recensement de 1981 (Statistički godišnjak Jugoslavije, 1990, p. 446-456), qui font état de 85000 Serbes et Monténégrins ayant quitté le Kosovo entre 1961 et 1981 et résidant dans le reste de la Yougoslavie, et de 9000 autres s'étant à l'inverse établis au Kosovo. Le solde migratoire, négatif, est donc de 76000 mais, compte tenu des décès survenus chez ces personnes entre la date de leur migration et celle du recensement, ce solde doit être réévalué à 84000 , et à près de 90000 si l'on tient compte de deux autres facteurs d'incertitude ${ }^{12}$.

19 Ces explications donnent à voir qu'on peut parfois démontrer le caractère exagéré de l'évaluation des migrations. Celui-ci se retrouve au début du XXI ${ }^{e}$ siècle où le nombre de Serbes et Monténégrins ayant fui le Kosovo est couramment évalué à 250000 (Dérens, 2005), alors qu'il en resterait 80000 à $100000^{13}$ dans la province et qu'en 1991 on n'en avait recensé que 215000 , population dont on a déjà montré qu'elle ne s'était probablement guère accrue dans les années 1990. De même, on peut soupçonner d'exagération (au moins initiale) le chiffre de 400 000, voire 500000 Albanais du Kosovo contraints de partir pour l'étranger au cours de années 1990 du fait du chômage de masse, de l'apartheid de fait et des violences policières en relevant que les milieux politiques albanais ont commencé à le répandre dès le début de la décennie et ne l'ont ensuite jamais révisé, comme si tout le monde était parti à la fois. Des Albanais ont qualifié cela de "nettoyage ethnique rampant " Jaka, 2002, p. 65). Par contre, les données démographiques sont floues: aucun recensement n'a eu lieu depuis 1991 au Kosovo et les données de l'état civil sont incomplètes : multiples naissances et décès non déclarés ou non enregistrés (Islami, 2005, p. 140). 
20 Outre l'exagération des chiffres, le discours politique et médiatique serbe sur l'émigration serbo-monténégrine du Kosovo expliquait celle-ci exclusivement par des pressions et des violences albanaises. Il est vrai qu'après les événements de 1981, les relations entre les deux communautés se sont dégradées. Dans un climat de peur réciproque, ce discours a été utilisé rétroactivement pour expliquer l'émigration des décennies précédentes. Sans vouloir nier ces tensions et l'existence d'incidents intercommunautaires, il faut rappeler que les départs avaient des causes essentiellement économiques, comme le soulignait entre autres un ouvrage de géographie (Rodić, 1986), et que si les Albanais étaient peu enclins à quitter le Kosovo pour d'autres parties de la Yougoslavie, c'est qu'il leur était beaucoup plus difficile qu'aux Serbes d'y trouver du travail et de scolariser leurs enfants dans leur langue maternelle. D'autre part, les départs étaient tout aussi fréquents dans les communes pauvres de Serbie du Sud, hors Kosovo, hors de toute présence albanaise.

21 Le point d'orgue du discours victimiste a sans doute été atteint en 1986 dans le Mémorandum de l'Académie des sciences de Serbie, document interne révélé par une «fuite» dans la presse et qui, après avoir critiqué sévèrement la politique des communistes vis-à-vis de la question nationale, accuse les Albanais de "génocide physique, politique, juridique et culturel de la population serbe au Kosovo-Metohija " (extraits dans Grmek et al., 1993). Il donne à réfléchir sur ce que peut être un " génocide » sans morts. En fait, ce document accuse les Albanais de pousser sournoisement les Serbes hors du Kosovo, et conclut que, sans un changement radical de politique, il n'y aura plus de Serbes au Kosovo dans une dizaine d'années et ce territoire sera "ethniquement pur, objectif clairement exprimé des racistes pan-albanais". Cette vision dramatisée se retrouve à la même époque chez l'historien Dimitrije Bogdanović, membre de cette même Académie : «La question ne peut être réduite au statut constitutionnel du Kosovo ni à la position des Albanais de Yougoslavie. C'est au contraire, pour bien plus de raisons, la question de la survie et de la position de la nation serbe tout entière, au Kosovo, en Yougoslavie, dans les Balkans » (Bogdanović, 1986, p. 259).

Un autre aspect du discours alarmiste serbe consistait à affirmer que les Albanais proliféraient à dessein, avec une "natalité africaine $»^{14}$. On en trouve une version élaborée dans un commentaire des résultats du recensement de 1991 dû à Ruža Petrović, professeur à l'Université de Belgrade :

Ce texte est typique du point de vue serbe dominant en ce sens qu'il érige en exception le cas des Albanais et impute le maintien d'une natalité élevée à un dessein politique. En fait, selon les données officielles, le taux de natalité des Albanais du Kosovo est passé de 51,2\%o (1950) à 42,1\%o (1970) et 30,5\%o (1990). Au-delà de cette date, il devient de plus en plus hasardeux de l'évaluer ${ }^{15}$. Il est vrai que, dans le même temps, le taux de natalité des Serbes du Kosovo est passé de 45,8 à 18,9, puis à 15,1. L'analyse qui vient d'être citée s'inscrit dans un courant qui considère la natalité des Albanais de toute l'ex-Yougoslavie comme anormalement élevée eu égard à leur degré de développement socio-économique. Cette "anomalie» est expliquée par l'influence conjuguée du modèle de la famille patriarcale albanaise, des milieux islamistes traditionalistes et du nationalisme albanais. En somme, il s'agirait de submerger les Serbes sous le nombre, du moins au Kosovo.

24 La réponse des intellectuels albanais se situait sur un autre terrain. Pour Hivzi Islami, géographe spécialiste des questions de population, recensements et enquêtes " témoignent des différences de taux de natalité et de fécondité entre les membres d'une même 
nationalité ou confession s'ils vivent dans des territoires inégalement développés du point de vue social et économique et s'ils n'ont pas la même position sociale, professionnelle et intellectuelle " (Islami, 1987), de sorte que les différences entre groupes nationaux relèvent d'abord de leurs structures socio-économiques et de leur niveau d'éducation, et seulement à titre secondaire de leurs caractéristiques nationales. Ces idées ne sont d'ailleurs pas propres aux Albanais. Elles furent accréditées, en des temps moins passionnés, par un important travail de recherche accompli au Centre d'études démographiques de l'Institut des sciences sociales de Belgrade sous la direction de Dušan Breznik (Rašević, 1971 ; Breznik et al., 1972 ; Rančić, 1973 ; Collectif, 1978). De 1961 à 1981, au Kosovo, le nombre moyen d'enfants nés vivants par femme a baissé plus vite chez les femmes actives (de 5,10 à 3,07) que chez les inactives (de 6,41 à 5,49) (Todorović, 1984-1985, p. 58). Selon le recensement de 1971, ce nombre était de 6,4 pour les femmes jamais scolarisées, de 1,9 pour celles qui avaient fait des études secondaires. En somme, c'est l'insuffisance du développement régional qu'il faut incriminer. D'ailleurs, tous les groupes nationaux présentaient au Kosovo une natalité plus élevée que dans le reste de la Yougoslavie. Avec 41,2 \%o en 1953, les Serbes y talonnaient les Albanais alors que l'ensemble des Serbes de Yougoslavie n'arrivait qu'à $28 \%$, et si leur déclin, ensuite, a été rapide (notamment en raison des départs de jeunes adultes), l'écart avec la moyenne serbe est resté longtemps important : 31,3 contre 19,3 en 1961, 22,4 contre 15,3 en 1971 (Collectif, 1978, p. 31). Singulière en Europe dans la seconde moitié du XX siècle, la dynamique démographique albanaise se situe, à l'échelle mondiale, dans le groupe des transitions démographiques longues, selon la typologie de Jean-Claude Chesnais (Chesnais, 1979).

Le discours serbe sur la prolifération albanaise comporte un sous-thème : la submersion territoriale du Kosovo. Alors que Ruža Petrović, déjà citée, parle de l'inertie migratoire des Albanais, car elle ne se réfère qu'aux migrations interrégionales, Branislav Krstić, dans une étude détaillée des dynamiques démographiques du Kosovo, s'intéresse aux migrations intercommunales dans la province et montre dans un chapitre intitulé " l'occupation ethnique des territoires " que, moins une commune comporte d'Albanais au départ, plus l'immigration albanaise y est intense (Krstić, 1994, chap.6). Il oublie simplement de rappeler que les communes presque exclusivement albanaises sont situées dans le rural "profond", tandis que les communes à population mixte sont celles qui hébergent les grandes villes, pôles d'industrialisation et de développement à l'époque qu'il étudie. Il s'intéresse ensuite aux changements interethniques de propriété (maisons, terres agricoles), thème si sensible que le régime de Milošević avait interdit aux Serbes de vendre aux Albanais.

L'argumentaire serbe comporte un autre thème: l'immigration albanaise aurait été massive depuis l'Albanie au début de l'après-guerre, portant sur plusieurs centaines de milliers de personnes ${ }^{16}$ que Tito aurait laisser entrer en Yougoslavie. "Il leur a tout donné, les maisons et les terres, et sans payer, et à nos dépens", entendait-on dire en substance dans le peuple, surtout chez les Serbes du Kosovo. Lors des manifestations de 1988 en faveur de ceux-ci, cet argument était utilisé. Le 23 septembre, on pouvait lire à Užice sur une pancarte : «Plus de 300000 émigrants venus d'Albanie ont occupé le couur de la Serbie. Message du peuple : dehors ». En fait, l'Albanie, qui n'avait guère plus d'un million d'habitants à la fin de la Seconde Guerre mondiale, ne pouvait pas à la fois exporter tant de gens et tripler sa population en quatre décennies. Les comportements analogues observables de part et d'autre de la frontière en matière de natalité expliquent au contraire une croissance presque parallèle du nombre d'habitants, avec 
un assez modeste apport migratoire de l'Albanie entre 1941 et 1948 . Ces allégations invraisemblables sont d'ailleurs contradictoires avec le thème de la prolifération in situ, mais peu importe, puisque leur objectif commun est de nourrir l'idée d'une inondation démographique, et que le second a l'avantage de représenter plus explicitement les Albanais comme des envahisseurs.

En résumé, la conception serbe courante de l'exception démographique albanaise n'est pas scientifiquement fondée. Elle témoigne seulement, dans un contexte de pathologie des rapports interethniques suscitée par des rivalités politiques fortes et un battage médiatique incessant, de l'angoisse ressentie par un peuple parvenu en fin de transition démographique et dont l'effectif amorce un déclin, face au dynamisme d'un voisin moins avancé dans le même processus et considéré par lui comme un concurrent, s'agissant du Kosovo. Il était désagréable aux Serbes de constater que les Albanais avaient assuré 80 \% de la croissance de la population de la République de Serbie entre 1981 et 1991. Et plus désagréable encore de penser qu'ils pourraient dépasser les Serbes, sur le plan du nombre, en moins d'un siècle en République de Serbie, et prendre ainsi le pouvoir par le bulletin de vote (toutefois, une prévision démographique à l'échéance d'un siècle n'a guère de sens). Cette perspective fournissait d'ailleurs un argument à ceux qui, dans les années 1990, se résignaient à envisager la perte du Kosovo, les deux autres arguments étant le coût budgétaire exorbitant de l'appareil répressif et l'effondrement de l'économie locale. Quant au Kosovo, Serbes et Monténégrins n'en constituaient plus, en 1991, que $11 \%$ de la population. Ils vivaient cette situation dans l'angoisse, se sentant marginalisés et craignant que la Serbie ne perde la région ${ }^{17}$. Au début du XXI ${ }^{\mathrm{e}}$ siècle, leur part est tombée aux environs de $5 \%$ et leur situation est devenue intenable.

\section{Les Serbes sont-ils en train de perdre le Kosovo? Déplacés et enclaves}

Serbes et Monténégrins sont, depuis l'été 1999, probablement moins de 100000 au Kosovo, ce qui signifie que largement plus de 100000 ont fui cette région ${ }^{18}$. Leur répartition géographique y revêt deux formes très différentes (figure 1). Dans le nord, qui est extérieur à l'aire de peuplement majoritairement albanais, ils constituent la presque totalité de la population des communes de Leposavić, Zubin Potok et Zvečan et d'une partie de la ville de Mitrovica, en rive gauche de l'Ibar ${ }^{19}$, environ 50000 personnes en tout. Leur mode de vie est normal, à ceci près que l'économie locale est sinistrée. Par contre, dans le reste du Kosovo, Serbes et Monténégrins vivent dans des enclaves protégées par les forces internationales et n'en sortent qu'en convois escortés. Malgré l'assistance que lui prodigue le gouvernement serbe, la vie quotidienne est plutôt sinistre pour une population vieillissante, en majorité privée d'emplois et tenaillée par un fort sentiment d'insécurité. Les principales enclaves, situées autour de Gračanica, Gnjilane, Vitina et Štrpce, comptent plusieurs milliers d'habitants chacune. Le sentiment d'enfermement et de précarité est plus fort encore dans les petites enclaves : quelques immeubles à Priština, un quartier clôturé dans la ville d'Orahovac, un village isolé comme Svinjare au sud de Mitrovica. Des reportages le soulignent en recourant à la métaphore de la prison (Despic-Popovic, 2004) ou de l'exil intérieur (Dubuisson et Davoine, 2005). Ces petites enclaves sont celles qui se dépeuplent le plus vite, d'autant plus que Belgrade ne les soutient guère mais concentre ses efforts sur les 
principales, espérant obtenir qu'elles soient détachées du Kosovo si la communauté internationale opte pour l'indépendance de celui-ci, et au minimum qu'elles soient dotées d'une autonomie très étendue.

Figure 1 : Localisation des minorités ethniques au Kosovo.

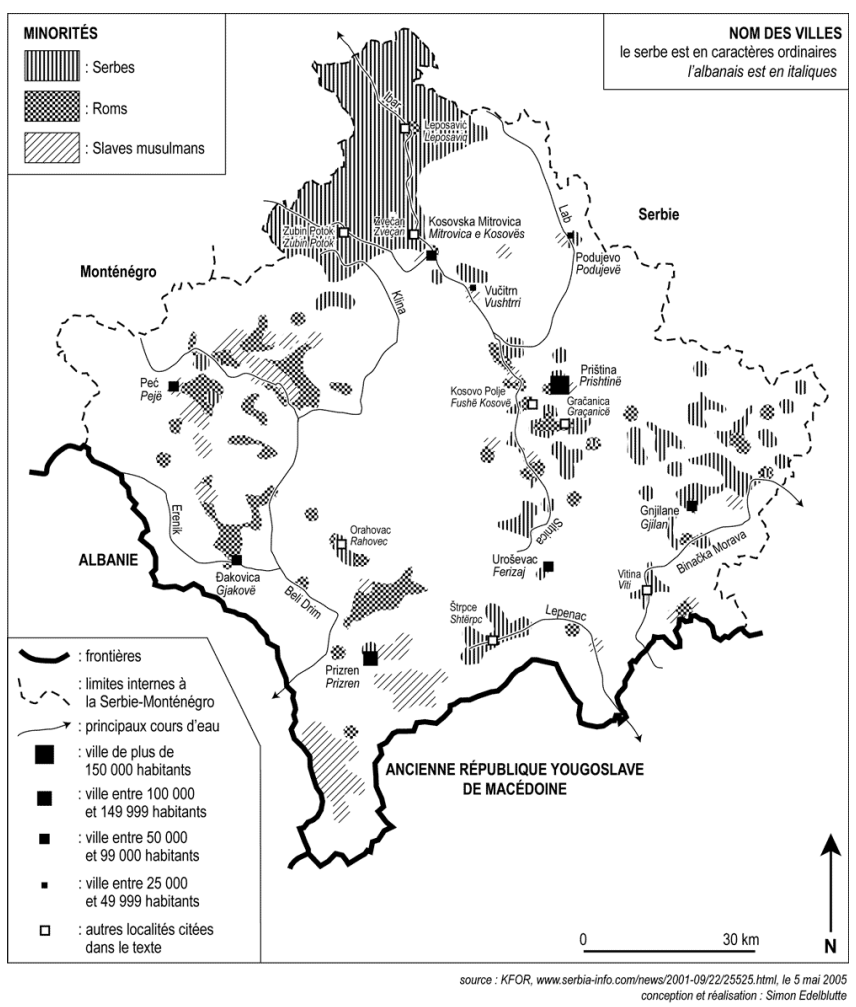

Cette ghettoïsation des Serbes (ainsi que des Roms) s'est imposée très rapidement pendant l'été 1999, lors du retour des Albanais auparavant expulsés et en dépit du déploiement des forces de l'OTAN et de la mise en place de la mission de l'ONU. À côté des expulsions, elle constitue un aspect d'un "nettoyage ethnique » qui, même s'il ne relevait pas du pouvoir organisé et des plans préconçus d'un appareil d'État, a été efficace. Ce nettoyage est dénoncé par les Serbes comme relevant d'un vieux projet albanais de "Kosovo ethniquement pur» appelé à faire partie d'une "Grande Albanie ", ainsi par l'ancien président de la République Fédérale de Yougoslavie ${ }^{20}$ Dobrica Ćosić (Ćosić, 2004). Cette dénonciation se nourrit d'ailleurs d'arguments puisés dans l'autre camp: l'universitaire kosovar Rexhep Qosja n'a-t-il pas publié un argumentaire passionné en faveur de l'union de tous les Albanais dans un même État, qu'il appelle "Albanie naturelle » - c'est-à-dire ethnique ? (Qosja, 1995). En face, ce "nettoyage" est présenté comme un ensemble de "vengeances perpétrées par des Albanais traumatisés par la guerre et l'exode» (Jaka, 2002, p.68). Cette explication minimaliste, en se référant aux réactions spontanées d'individus ordinaires, a l'intérêt de souligner la profondeur de la haine qui s'est instaurée et de montrer quel obstacle elle constitue à la pacification et à la démocratisation du Kosovo. Mais elle passe sous silence la part de violence organisée imputable aux fractions les plus dures de l'UÇK pour lesquelles moins il restera de Serbes et de signes de leur présence passée dans le Kosovo, plus celui-ci aura de chances d'obtenir l'indépendance : stratégie d'effacement. Même après la dissolution officielle de cette organisation, des réseaux d'activistes subsistent; on sait leur rôle dans l'insurrection albanaise de Macédoine au printemps 
2001, on le soupçonne dans la flambée de violence survenue en mars 2004 : suite à la noyade de deux enfants albanais dans l'Ibar, imputée à des Serbes, des agressions simultanées contre les enclaves serbes, dans tout le Kosovo, avaient causé en deux jours la mort d'une vingtaine de personnes, l'incendie d'environ 800 maisons, et amené les forces internationales, elles-mêmes molestées, à héberger provisoirement sur leurs bases plusieurs milliers de Serbes. Il y a là, par le biais de l'exploitation émotionnelle d'un événement violent (lui-même précédé du meurtre de trois Serbes), une probable tentative de susciter de nouveaux départs et de décourager des retours, et en tout cas de faire échouer les entretiens qui venaient de débuter entre représentants kosovars et gouvernement serbe sur la question des disparus de 1999 (ces entretiens ont repris un an plus tard). Cette tentative a bien été identifiée comme telle par la MINUK et par les médias occidentaux, même si sa portée a parfois été exagérée, ainsi lorsque le journal La Croix titrait « Kosovo : les Albanais chassent les derniers Serbes » (D'Alançon, 2004).

Bref, tout semble confirmer qu'aucune détente véritable ne se dessine au Kosovo entre Serbes et Albanais, même si l'on a noté que des notables des deux bords skiaient ensemble à Brezovica, dans l'enclave serbe de štrpce. Il est vrai qu'après la décomposition violente de l'ex-Yougoslavie, l'apaisement demande du temps. Ainsi, en Croatie, un sociologue qui étudie à la loupe, depuis plus de dix ans, sur l'un des théâtres de la guerre les rapports entre Croates locaux (chassés en 1991, revenus en 1995), Serbes locaux (vainqueurs en 1991, chassés en 1995, partiellement de retour) et Croates réfugiés de Bosnie, commence à noter le rétablissement des liens sociaux primaires et en conclut que le conflit n'était pas interethnique mais politique (Babić, 2004). Mais on peut craindre qu'entre Serbes et Albanais une telle évolution soit presque impossible. Ces deux groupes, d'abord, n'ont jamais dépassé le stade d'une cohabitation quelque peu distante pendant les meilleures périodes (quasi-absence de villages mixtes et de mariages mixtes, par exemple). Le fossé entre eux s'est élargi de nouveau à partir de 1981 et une nouvelle difficulté est apparue dans les années 1990 : les jeunes Albanais, exclus de l'enseignement public et éduqués dans un système scolaire parallèle, ne savent pas le serbe ou le savent à peine, contrairement à leurs aînés, alors que très peu de Serbes parlent albanais. Au-delà des préventions et des haines réciproques, il y a là un obstacle à un hypothétique rapprochement. En second lieu, la question du statut politique définitif du Kosovo, sur laquelle ils ont des positions inconciliables, n'est pas résolue. Surtout, l'accumulation de violences qui caractérise leur histoire commune au $\mathrm{XX}^{\mathrm{e}}$ siècle marque les mémoires, probablement plus que partout ailleurs dans les Balkans, outre les griefs croisés qui émergent d'interprétations contradictoires de l'histoire des siècles précédents, asservies aux nationalismes. Ce genre de discours exporte ordinairement la responsabilité des violences sur le camp adverse et exagère le nombre de victimes de son propre camp. À tel point que, si le nombre d'expulsés revendiqué de part et d'autre tout au long du XXe siècle était exact, le Kosovo serait pratiquement désert. En fait, le "nettoyage ethnique » a joué dans la marginalisation des Serbes et des Monténégrins au Kosovo, jusqu'en 1999, un rôle secondaire par rapport à celui des processus démographiques ordinaires, accroissement naturel et migrations économiques. Mais il aurait pu entraîner celle des Albanais si en 1999 Milošević l'avait emporté, c'est-à-dire si la communauté internationale n'était pas intervenue. Et il a, en fin de compte, parachevé celle des Serbes et des Monténégrins. Par ailleurs, la diabolisation des processus démographiques ordinaires dans le discours serbe a accru l'incompréhension et l'hostilité entre les deux peuples. De même, la revendication albanaise d'une septième république yougoslave (1981), quand le Kosovo 
fonctionnait déjà comme une quasi-république, était probablement une erreur tactique propre à générer le soupçon de séparatisme ; le refus de tout dialogue et la répression violente qui ont suivi en étaient également une. À partir de là, tout a été interprété comme relevant de la volonté d'éliminer l'Autre du Kosovo : le "nettoyage ethnique ", ou la volonté de l'accomplir, étaient vus partout. L'échec de l'ex-Yougoslavie est de ne pas avoir su instaurer des conceptions et des pratiques civiques qui auraient mis fin à ce qui était vécu de part et d'autre comme une compétition pour la possession exclusive de ce territoire.

Depuis 1999, l'ONU défend l'idée d'un « Kosovo multiethnique» sans pouvoir la faire entrer dans les faits, d'autant plus qu'" en protégeant la minorité serbe, l'OTAN entérine sans le vouloir la division ethnique du Kosovo » (Zecchini, 2004) et que cette minorité, frustrée et influencée par les courants nationalistes radicaux de l'échiquier politique de Belgrade, refuse de jouer le jeu proposé par l'ONU, celui de mettre en place une bonne gouvernance du Kosovo en attendant la solution définitive (abstention lors des élections législatives d'octobre 2004, refus de siéger des élus déjà auparavant). Du point de vue de l'ONU, le Kosovo serait donc en phase d'apprentissage (de la démocratie, d'une multiethnicité assumée). Pour d'autres, l'absence prolongée de perspectives claires sur le statut définitif de la région est la principale source de tensions et de violences (Crémieux, 2004). Le chef de la Mission de l'ONU au Kosovo estime ${ }^{21}$ que cette question a de bonnes chances d'être abordée au second semestre de 2005. Mais c'est une question épineuse pour au moins quatre sortes de raisons: Serbes et Albanais du Kosovo ont des options incompatibles sur ce sujet ; l'union Serbie-Monténégro, forme actuelle de la Yougoslavie dont la résolution 1244 du Conseil de sécurité de l'ONU reconnaît que le Kosovo fait partie, est menacée de dissolution à court terme par le séparatisme monténégrin, événement qui, s'il se produisait, poserait le problème juridique de la succession de cet État et stimulerait l'indépendantisme kosovar; la perspective d'une proclamation unilatérale de l'indépendance de la Republika Srpska, entité serbe de Bosnie, peut être utilisée comme un contre-feu par rapport à l'idée de l'indépendance du Kosovo; celui-ci est économiquement sinistré et traversé de multiples trafics illégaux. Se verra-t-il attribuer une indépendance totale, une indépendance conditionnelle et progressive à la mesure de ses progrès en matière de démocratie et de respect des droits de l'homme et des minorités, une autonomie très étendue dans le cadre de la Serbie-Monténégro ou de la Serbie seule ? Dans ses limites actuelles ou après redécoupage sur critères ethniques? Ou bien demeurera-t-on indéfiniment dans le statu quo actuel ? Il s'agit du problème le plus difficile sur la voie de l'apaisement des Balkans occidentaux et de leur intégration à l'Union européenne. $\mathrm{Au}$ bout du compte, il semble que le "nettoyage ethnique» ait joué un rôle numériquement secondaire sur le long terme, mais finalement décisif, en se retournant contre ses promoteurs pour parachever l'albanisation du Kosovo ${ }^{22}$. Il est fort improbable qu'un nombre significatif de Serbes et de Monténégrins y revienne, même si les dirigeants albanais locaux les y invitent officiellement ${ }^{23}$. Toujours surpeuplé et sans perspectives de développement, il devrait demeurer dans les prochaines décennies, comme d'autres terres balkaniques, un foyer d'émigration économique à l'échelle européenne et mondiale, quelque soit son statut politique définitif. À court terme, l'organisation d'un recensement de la population répondant aux normes internationales y serait d'une grande utilité. 


\section{BIBLIOGRAPHIE}

BABIĆ D. (2004). - « Suzivot Hrvata I Srba u prijeratnom, ratnom i poslijeratnom razdoblju implicitna kritika interpretacije rata u Hrvatskoj kao etnickog sukoba », [La coexistence des Croates et des Serbes avant, pendant et après la guerre, critique implicite de l'interprétation de la guerre en Croatie comme conflit ethnique], Migracijske i etnicke teme, $\mathrm{n}^{\circ}$ 2-3, pp. 187-208.

BATAKOVIĆ D. (2000). - « Kosovo à l'époque titiste : entre nationalisme et communisme ». In :

Kosovo, six siècles de mémoires croisées, Les Annales de l'autre Islam, Paris, INALCO-ERISM, $\mathrm{n}^{\circ} 7$, pp. 205-224.

BOGDANOvić D. (1986). - Knjiga o Kosovu [Livre sur le Kosovo], Belgrade, Académie serbe des sciences et des arts, $297 \mathrm{p}$.

BRAEM Y. (2004). - « Mitrovica/Mitrovicë. Géopolitique urbaine et présence internationale », Balkanologie, VIII, $\mathrm{n}^{\circ}$ 1, pp. 73-104.

BREZNIK D. et al. (1972). - Fertilitet stanovnistva u Jugoslaviji [La fécondité de la population en Yougoslavie], Belgrade, Centre d'études démographiques.

CHESNAIS J.-C. (1979). - « L'effet multiplicatif de la transition démographique », INED, Population, $\mathrm{n}^{\circ}$ 6, pp. 1138-1144.

Collectif (1953). - Stanovnistvo NR Srbije od 1834-1953 [Population de la République Populaire de Serbie de 1834 à 1953], Belgrade, Institut de statistique de Serbie.

Collectif (1978). - Demografska kretanja i karakteristike stanovnistva Jugoslavije prema nacionalnoj pripadnosti [Évolution et caractéristiques démographiques de la population de la Yougoslavie selon l'appartenance nationale], Belgrade, Centre d'études démographiques.

ćosić D. (2004). - « Kompromis za vekovni antagonizam » [Compromis pour un antagonisme séculaire], Belgrade, interview à Vecernje Novosti, 21/10/2004.

CRÉMIEUX F. (2004). - « Kosovo : vite, le pouvoir aux Albanais! », Le Monde, $1^{\mathrm{er}}$ avril.

D’ALANçon F. (2004). — « Kosovo : les Albanais chassent les derniers Serbes », La Croix, 19 mars.

DÉRENS J.-A. (propos recueillis par) (2005). - « Sans le retour des Serbes la reconstruction n'est pas possible ", entretien avec Mgr Artemije, évêque de Prizren et de Raska, La Croix, 5 janvier.

DESPIC-POPOVIC H. (2004). - « Les Serbes du Kosovo vivent comme dans une prison », Libération, 25 octobre, p. 10 .

DJURIC I. (1999). - Glossaire de l'espace yougoslave, Paris, l'Esprit des Péninsules, article « Kosovo », pp. 85-92.

DRAGNICH A., TODOROVICH S. (1984). - The Saga of Kosovo. Focus on Serbian-Albanian Relations, Boulder, East European Monographs, distribué par Columbia University Press, New York, 203 p.

Dubuisson A., DAvoinE J. (2005). - « L'exil intérieur des minorités kosovars ». Convergence, Secours Populaire Français, janvier, pp. 12-13.

DuIzJINGS G. (2000). - Religion and the Politics of Identity in Kosovo,Londres, Hurst \& Co., 238 p.

ESI (European Stability Initiative). (2004). - The Lausanne Principe : Multiethnicity, Territory and the Future of Kosovo's Serbs, Berlin - Pristina. 
GRMEK M. et al. (1993). - Le nettoyage ethnique. Documents historiques sur une idéologie serbe, Paris, Fayard, pp. 236-269.

GRAVIER G. (1913). - « L'Albanie et ses limites », Revue de Paris, 1-15 janvier.

HUBRECHT J. (2001). - Kosovo 1981-1989-1999-2001. Établir les faits, Paris, Éditions Esprit, 176 p.

IsLAmi H. (1987). - « Problèmes de la natalité chez les Albanais », Recherches albanologiques, $\mathrm{n}^{\circ} 4$.

IsLAMI H. (2005). - Studime demografike. 100 vjet të zhvillimit demografik të Kosovës [Études démographiques. Cent ans de développement démographique du Kosovo], Prishtinë, Académie des sciences et des arts du Kosovo, $534 \mathrm{p}$.

JAKA Y. (2002). - « La guerre et les nettoyages ethniques au Kosovo ». In : Lepage D. et Kullashi M. (dir.), Ex-Yougoslavie : une Europe du Sud-Est en construction, Paris, L’Harmattan, pp. 61-72.

KRSTIĆ B. (1994). - Kosovo izmedu istorijskog i etničkog prava [Le Kosovo entre droit historique et droit ethnique], Belgrade, Kuća vid, 320 p.

KULLASHI M. (1998). - Humanisme et haine. Les intellectuels et le nationalisme en ex-Yougoslavie, Paris, L'Harmattan, $206 \mathrm{p}$.

LUKIĆ R. (2003). - L'agonie yougoslave (1986-2003), Québec, Les presses de l'Université Laval, 613 p. OBRADOVIĆ M. (1981). - Agrarna reforma i kolonizacija na Kosovu (1918-1941) [Réforme agraire et colonisation au Kosovo, 1918-1941], Pristina, Institut d'histoire du Kosovo, 357 p.

OSTRENI A. (1971). - Gjeografia e Shqipërisë për shkollat e mesme [Géographie de l'Albanie pour les écoles moyennes], Tirana, Shtëpia botuese e librit shkollor, $224 \mathrm{p}$.

PETROvić R. (1992). - « The National Composition of Yugoslavia's Population », Yugoslav Survey, XXXIII, $\mathrm{n}^{\circ} 1$, pp. 3-24.

Programmes of the Parliamentary Political Parties (1993). - Belgrade, Yugoslav Survey, XXXIV, $\mathrm{n}^{\circ} 1$, pp. 51-106.

QOSJA R. (1995). - La question albanaise, Paris, Fayard, 326 p.

RANĆIĆ M. (1973). - Fertilitet autohtonog i migrantskog stanovništva u Jugoslaviji [Fécondité de la population autochtone et de la population migrante en Yougoslavie], Belgrade, Centre d'études démographiques.

RAŠEVIĆ M. (1971). - Determinante fertiliteta stanovništva Jugoslavije [Les déterminants de la fécondité de la population de la Yougoslavie], Belgrade, Centre d'études démographiques.

Recnik mesta u oslobodenoj oblasti Stare Srbije [Registre des lieux dans les territoires libérés de Vieille Serbie]. - Belgrade, 1914.

RODIĆ D. (1986). - Geografija Jugoslavije, Belgrade, Naucna knjiga, 2 vol., 277 + 432 p.

Roux M. (1997). - « Serbes et Albanais au Kosovo », Slavica Occitania, n 3, pp. 87-106.

Roux M. (2000). - « Enjeux démographiques au Kosovo ». In : Kosovo, six siècles de mémoires croisées, Les Annales de l'autre Islam, Paris, INALCO-ERISM, n 7, pp. 121-144.

Statisticki godisnjak Jugoslavije [Annuaire statistique de la Yougoslavie]. - Belgrade, Institut fédéral de statistique, éditions 1955 à 1991.

TODOROVIĆ G. (1984-1985). — « Dejstvo aktivnosti i obrazovanja zene na njeno radanje i stav o njemu na podrucjima SR Srbije » [Influence de l'activité et de l'éducation des femmes sur leur fécondité ; situations régionales en République de Serbie], Stanovnistvo, n 1-4, p. 58. 
ZECCHINI L. (2004). - « En protégeant la minorité serbe, l'OTAN entérine sans le vouloir la division ethnique du Kosovo », Le Monde, 7 décembre.

\section{NOTES}

1. Il s'agit du Kosovo dans sa définition territoriale actuelle, due au régime de Tito. Rappelons que le nom de Kosovo Polje (Champ des Merles) désignait à l'origine le lieu de la bataille que se livrèrent Serbes et Turcs en 1389. Par extension, on a appelé Kosovo la totalité de la plaine qui s'étend de Mitrovica à l'amont des gorges de Kačanik. La Yougoslavie fédérative institua en 1945 dans le cadre de la République de Serbie une région autonome nommée Kosovo-Metohija (cette dernière étant l'autre plaine, plus proche de la frontière de l'Albanie), en abrégé Kosmet, puis simplifia ce nom en Kosovo. Slobodan Milošević revint au nom double, toujours utilisé par les Serbes alors que les Albanais disent Kosovë (au féminin dans leur langue).

2. Le cas de ces deux populations sera traité conjointement, du fait de leurs fortes affinités identitaires, de leurs solidarités et de leurs dynamiques communes dans le cadre de la question du Kosovo.

3. Environ $65 \%$ en 1905 (Djuric, 1999, p. 86).

4. La Constitution distinguait des nations, constitutives de l'État yougoslave, et des nationalités, euphémisme pour minorités.

5. Cette même revendication, en 1968, avait entraîné une répression mais aussi, probablement, inspiré à Tito l'idée d'accroître l'autonomie des provinces dans le contexte d'un processus général de décentralisation et de promotion de l'autogestion.

6. Dénombrement opéré par l'armée serbe (Rečnik mesta, 1914), complété pour la partie dévolue au Monténégro par une estimation (Collectif, 1953).

7. Exemple : un manuel de géographie publié en Albanie en 1971 attribue au Kosovo 1,2 million d'habitants albanais, surestimation délibérée d'un tiers par rapport aux résultats du recensement yougoslave de la même année (Ostreni, 1971, p. 99).

8. Certaines sources mentionnent jusqu'à 20000 réfugiés. Toutefois, des Serbes ayant fui la Krajina ou la Bosnie répugnaient à se retrouver face à des Albanais dont ils avaient une image totalement négative, et ne pensaient le plus souvent qu'à quitter la région.

9. En juin 1999, le HCR estimait à 980000 le nombre des expulsés décomptés depuis mars 1998, dont 443000 en Albanie, 249000 en Macédoine, 68000 au Monténégro, 22000 en Bosnie, 198000 dans d'autres pays.

10. Le Point, 14/4/1986, p. 93 ; Libération, 7/2/1987 et 23/12/1988.

11. Večernje Novosti, 11/12/1990, p. 6 .

12. D'une part l'absence de réponse précise de certaines personnes recensées à la question sur les migrations intérieures, d'autre part la «perte » statistique de certains émigrés à l'étranger (l'ex-Yougoslavie considérait ses émigrés économiques à l'étranger comme temporaires et les recensait dans sa population légale... sauf ceux dont personne n'avait plus de nouvelles).

13. Chiffre donné par Gojko Savić, membre de la présidence du Parlement du Kosovo, Danas, 11/4/2003. Dans le même entretien, il parle de 200000 Serbes déplacés.

14. Expression relevée dans le quotidien Politika, 30/10/1989, p. 9.

15. Le bulletin de l'Institut de statistique du Kosovo, Sistemi $i$ statistikave vitale, $\mathrm{n}^{\circ} 1$, décembre 2002, donne pour 2002 une moyenne mensuelle de 3495 naissances. Les communes serbes du Kosovo n'ayant pas communiqué leurs données, on peut estimer qu'au moins $95 \%$ de ces naissances sont albanaises, soit environ 40000 sur une année (à comparer à environ 48000 en 1990). Mais à quelle population rapporter ce nombre? Depuis 1990, beaucoup d'Albanais ont 
émigré. Les services statistiques du Kosovo sont-ils informés des naissances survenues à l'étranger?

16. Jusqu'à 700 000. Entendu à Vučitrn en 1992.

17. Ainsi, le 20 décembre 1992, jour d'élections législatives et présidentielles, des Serbes de Vučitrn m'ont longuement exprimé leurs angoisses et leurs griefs : "nous ne sommes plus que $3 \%$ de Serbes dans cette ville, la rue principale est passée aux Chiptars [dénomination des Albanais reprenant le mot albanais Shqiptar (" Albanais »), mais péjorative en serbe] maison après maison, ces lâches attendent que l'Europe leur donne un État, nous sommes dos au mur, mais nous avons des armes et nous combattrons ".

18. Un rapport de l'European Stability Initiative estime toutefois qu'en 2004 les Serbes seraient $130000 \mathrm{au}$ Kosovo (ESI, 2004).

19. Sur la situation de cette ville coupée en deux, voir Yann Braem (2004).

20. Aujourd'hui Serbie-Monténégro.

21. Dans son rapport présenté au Conseil de sécurité le 24 mars 2005.

22. À ceci près que cette région comporte, rappelons-le, trois autres minorités notables, Roms, Musulmans (slavophones) et Turcs, en tout 6,4 \% de sa population en 1981, 6,2\% en 1991. Les deux dernières sont dans une large mesure demeurées sur place.

23. Les principaux dirigeants politiques du Kosovo ont signé le $1^{\mathrm{er}}$ juillet 2003 une déclaration invitant les Serbes à revenir. À cette date, le HCR estimait que 4000 retours avaient déjà eu lieu. Institute for War and Peace Reporting, 4/7/2003.

\section{RÉSUMÉS}

Bien que depuis 1912 le Kosovo soit resté dans le cadre de la Serbie et du Monténégro, puis des Yougoslavie successives (sauf de 1941 à 1945) et sous le contrôle effectif de leurs gouvernements (sauf de 1915 à 1918 et depuis 1999), Serbes et Monténégrins ont vu leur part dans sa population diminuer jusqu'à ne plus en constituer que $5 \%$ tandis que la majorité albanaise dépasse les $90 \%$. Cela s'explique par un ensemble de causes se combinant différemment selon les périodes: différences d'accroissement naturel, migrations spontanées, modifications coercitives du peuplement, implantation de colons. Déterminer la part réelle du «nettoyage ethnique » dans cette évolution implique de décrypter deux discours nationalistes antagonistes qui tendent à l'ériger en explication centrale de tous ces processus.

Although Kosovo belonged to the separate states of Serbia and Montenegro, then to the successive Yugoslav states (except from 1941 to 1945) and was under their effective control (except from 1915 to 1918 and since 1999), the proportion of Serbs and Montenegrins in its population diminished so that at the present time they form only $5 \%$ of it, while the Albanian majority exceeds $90 \%$. This can be explained by several causes in various combinations according to the periods: differences in natural growth, spontaneous migrations, forced migrations, colonization. To determine the actual role of ethnic cleansing in this evolution, it is necessary to decipher two opposite nationalistic discourses which try to make it the main explanation for all these processes.

Obwohl der Kosovo seit 1912 im Rahmen von Serbien und Montenegro, dann der Folgeformen von Jugoslawien (außer während der beiden Weltkriege) verblieben ist, ging der Anteil der 
Serben und Montenegriner gegenüber einer albanischen Mehrheit auf $5 \%$ zurück, obwohl es die Absicht der Regierung war, ein gewisses Gleichgewicht zu erreichen. Diese Entwicklung erklärt sich durch ein Bündel von Ursachen, die sich je nach Periode verschieden kombinieren: Unterschiede des natürlichen Wachstums, spontane Migrationen, zwangsläufige Modifikationen der Bevölkerung, Ansiedlung von Kolonisten. Den wahren Anteil der ethnischen Säuberung in dieser Entwicklung zu ermitteln schließt ein, zwei nationalistische und antagonistische Diskurse zu entziffern, die dazu tendieren, diese Säuberung zur zentralen Erklärung dieser Prozesse zu erheben.

\section{INDEX}

Schlüsselwörter : Balkan, Demographie, ethnische Säuberung, Kosovo, Migration

Keywords : Balkans, demography, ethnic cleansing, Kosovo, migration

Mots-clés : Balkans, démographie, Kosovo, migration, nettoyage ethnique

\section{AUTEUR}

\section{MICHEL ROUX}

UMR 5045 CNRS, Mutations des territoires en Europe, Département de Géographie, Université de Toulouse-Le Mirail, 5 allées Antonio Machado, F-31058 Toulouse cedex 9, France, mroux@univtlse2.fr 